\title{
United3D: A Protein Model Quality Assessment Program That Uses Two Consensus Based Methods
}

\author{
Genki Terashi, Makoto Oosawa, Yuuki Nakamura, Kazuhiko Kanou, ${ }^{\dagger}$ and \\ Mayuko Takeda-Shitaka* \\ School of Pharmacy, Kitasato University; 5-9-1 Shirokane, Minato-ku, Tokyo 108-8641, Japan. \\ Received March 30, 2012; accepted August 11, 2012
}

In protein structure prediction, such as template-based modeling and free modeling (ab initio modeling), the step that assesses the quality of protein models is very important. We have developed a model quality assessment (QA) program United3D that uses an optimized clustering method and a simple $\mathrm{C} \alpha$ atom contactbased potential. United3D automatically estimates the quality scores (Qscore) of predicted protein models that are highly correlated with the actual quality (GDT_TS). The performance of United3D was tested in the ninth Critical Assessment of protein Structure Prediction (CASP9) experiment. In CASP9, United3D showed the lowest average loss of GDT TS (5.3) among the QA methods participated in CASP9. This result indicates that the performance of United3D to identify the high quality models from the models predicted by CASP9 servers on 116 targets was best among the QA methods that were tested in CASP9. United3D also produced high average Pearson correlation coefficients $(0.93)$ and acceptable Kendall rank correlation coefficients (0.68) between the Qscore and GDT_TS. This performance was competitive with the other top ranked QA methods that were tested in CASP9. These results indicate that United3D is a useful tool for selecting high quality models from many candidate model structures provided by various modeling methods. United3D will improve the accuracy of protein structure prediction.

Key words protein structure prediction; model quality assessment; modeling; clustering; Critical Assessment of protein Structure Prediction

When experimental three dimensional (3D) structures of proteins are not available, protein structure prediction using computer is widely used in biology and medical research and has proven to be a useful tool. In the ninth community-wide experiment on the Critical Assessment of protein Structure Prediction (CASP9) ${ }^{1)}$ protein structure prediction methods, including the methods of 79 individual servers, were tested. ${ }^{2)}$ Protein structure prediction methods are still being developed and a lot of progress has been made in their accuracy. However, different prediction methods produce different candidate models for the same amino acid sequence. Clearly, the usefulness a predicted protein model depends on its accuracy, but the accuracy of the model cannot be ascertained when the experimental protein structure is unknown. To estimate the accuracy of predicted models when the experimental structure is not available, computational biologists have developed various quality assessment (QA) methods. The results of previous CASP experiments revealed that the models predicted as best by these methods are not always the models that are closest to the experimental protein structure. ${ }^{3-6)}$

The computational biology community, therefore, has focused their attention on the problem of how to estimate the quality of models without experimental structures. CASP established a QA category to test the performance of QA meth-

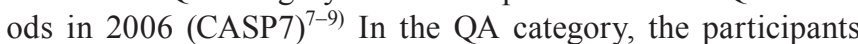
estimate the accuracy of protein models predicted by the tertiary structure prediction servers using their QA methods. The QA methods were assessed based on two criteria: (1) the correlation between the "estimated quality" (Qscore) predicted by

The authors declare no conflict of interest.

†Present address: Infectious Disease Surveillance Center, National Institute of Infectious Diseases; 1-23-1 Toyama, Shinjuku-ku, Tokyo 162-8640, Japan.
QA method and the "actual quality" (GDT_TS), and (2) their ability to select the best quality models from a set of predicted protein structures.

Accurate QA methods that are based on the coordinates of predicted models will contribute to the improvement of protein structure prediction strategies. Moreover, estimates of differences of the models from the native structure are useful to end users who can then decide on the usability of the models for their specific problems. Therefore, an accurate QA method is an essential component of protein structure prediction when multiple protein structure prediction methods and models are available. Existing QA methods can be divided into three categories according to the scoring functions that are used: (1) knowledge-based potential, ${ }^{10-13}$ (2) structural energies using molecular mechanics force fields, ${ }^{14)}$ and (3) clustering method. $^{15-17)}$

Based on earlier tests, the clustering method has been shown to outperform other methods. For example, the QA methods that were tested in the previous CASP experiments, $\mathrm{CASP}^{7}{ }^{7}$ (Pcons) ${ }^{18)}$ and CASP8 ${ }^{8)}$ (Pcons Pcons, ${ }^{19)}$ ModFoldClust $^{20)}$ and SAM-T08-MQAC) ${ }^{21)}$ all used the clustering method in their scoring functions. The clustering methods use a number of models and structurally compare them to calculate the consensus value. These methods use similar strategies based on the assumption that the model that has the greatest structural similarity to all other models (i.e., the model in the centroid of the cluster) will be the model that is structurally similar to the native structure. However, as the previous CASP experiments showed, the clustering method usually failed to identify the best models that were closest to the native structure.

Here, we describe a QA program United3D that we have developed based on optimized clustering methods. United3D employs two types of clustering-based scoring functions calculated from local and global structural similarities and 
considering only the $\mathrm{C} \alpha$ coordinates of the models. We considered that the ability to identify the best model from a set of models predicted by a number of different prediction methods was much more important for QA than the linear correlation measured by Pearson's correlation coefficient (Pearson's r). Therefore, United3D was optimized based on the Kendall rank correlation coefficient (Kendall's $\tau$ ) to estimate the quality of the models and to select the best model. To evaluate the performance of United3D, we participated in the QA category of CASP9. For the official CASP assessment, the quality of a model was evaluated by assigning it a Global Distance Test Total Score (GDT TS). The GDT TS is the number of residues in the predicted models that deviate from the native structure after four different sequence-dependent superpositions using $\mathrm{C} \alpha$ distance cut offs of $1,2,4$ and $8 \AA$. In this article, the "best model" represents the model which has the highest GDT_TS value (i.e., the closest to the native) for the set of models to be evaluated.

In the realistic view point, the situation in which various servers produce a large number of models at the same time, such as in the CASP experiments, is not the usual situation for a lot of researchers who employ QA methods to obtain accurate models or to know the usability of a model. To identify the applications of our method in a more realistic situation, we further examined the performance of United3D in a situation in which a limited number of models were available.

\section{Experimental}

The QA method in United3D is based on two types of quality score: (1) the consensus of the $\mathrm{C} \alpha$ position in the global structure $\left(S_{\text {global }}\right)$; and (2) the consensus of residue-residue contact in the structure $\left(S_{\text {local }}\right)$. Thus, $S_{\text {global }}$ and $S_{\text {local }}$ are based on the whole chain similarity considering the global structure and the contact similarity in the local structure, respectively. When the consensus values were calculated, to avoid redundancy of models, the model to be assessed was compared only with the first models (TS1 models) that were estimated as best by each of the testing structure prediction servers that was participating in CASP9. This procedure is similar to the 3DJury method. ${ }^{15)}$ In the present study, to describe the protein $3 \mathrm{D}$ structure the $\mathrm{C} \alpha$ coordinates of the amino acid residues were used as the $3 \mathrm{D}$ coordinates for each of the residues.

Consensus of the $\mathrm{C} \alpha$ Positions in Global Structures The quality score based on the consensus of the $\mathrm{C} \alpha$ positions was calculated by comparing the $\mathrm{C} \alpha$ positions in the model to be assessed with the $\mathrm{C} \alpha$ positions in the set of other models. As mentioned above, we used only the TS1 models predicted by each server as the set of other models. The structural similarity between two models $\left(M_{i}\right.$ and $\left.M_{j}\right)$ was represented as $\operatorname{SIM}_{d}\left(M_{i}, M_{j}\right)$ in the range 0 to 1 , where $d$ is the threshold of the distance between corresponding $\mathrm{C} \alpha$ pairs. This similarity is equal to the fraction of the maximum number of equivalent $\mathrm{C} \alpha$ atoms for which the distance between them is smaller than $d \AA$ after optimal superimposition of the corresponding residues on the target structure.

$S_{\text {global }}$ is described as follows,

$$
S_{\text {global }}=1-\sqrt[\text { pow }]{\frac{\sum_{i \in N}\left(1-\operatorname{SIM}_{d}\left(M_{\text {model }}, M_{i}\right)\right)^{\text {pow }}}{N}}
$$

where $N$ is the number of first models predicted by each server, and $M_{\text {model }}$ is the model to be assessed. The optimized parameters are $d=3.5$ and pow $=2.0$. Equation 1 shows that a model that has many regions that are similar to other models will have a high $S_{\text {global }}$. Thus, $S_{\text {global }}$ uses the structural consensus among the set of first models to estimate model quality.

Consensus of Residue-Residue Contact in Local Structures A pair of two residues was defined as being in residue-residue contact if the distance between their $\mathrm{C} \alpha$ atoms was less than $6 \AA$. $S_{\text {local }}$ is described as follows,

$$
\begin{aligned}
S_{\text {local }}= & \sum_{i \in L} \sum_{j \in L, i<j}\left(w_{\text {contact }} \cdot \log \left(\frac{P(i, j)}{P(|i-j|)}\right)\right. \\
& \left.+w_{\text {noncontact }} \cdot \log \left(\frac{P^{*}(i, j)}{P^{*}(|i-j|)}\right)\right)
\end{aligned}
$$

where $L$ is the length of a target sequence, $P(i, j)$ represents the fraction of models whose $i$-th residue is in contact with the $j$-th residues among the set of first models, and $P^{*}(i, j)$ represents the fraction of models whose $i$-th residue is not in contact with the $j$-th residue. In other words, $P^{*}(i, j)$ represents the fraction of models in which the distances between the $i$-th and $j$-th residues were greater than $6 \AA . P(|i-j|)$ and $P^{*}(|i-j|)$ are the fraction of contacting and non-contacting residue pairs, respectively, that are separated by $|i-j|$ residues in all possible residue pairs separated by $|i-j|$ residues. If $|i-j|$ was more than 20 , then $P(|i-j|)$ and $P^{*}(|i-j|)$ were set to $P(|20|)$ and $P^{*}(|20|)$. $w_{\text {contact }}$ and $w_{\text {noncontact }}$ are weight functions defined as follows,

$$
\begin{aligned}
w_{\text {contact }} & = \begin{cases}1 & \text { if } d(i, j) \leq 6.0 \text { and } P(i, j) \geq 0.3 \\
0 & \text { otherwise }\end{cases} \\
w_{\text {noncontact }} & = \begin{cases}1 & \text { if } d(i, j)>6.0 \text { and } P(i, j) \geq 0.3 \\
0 & \text { otherwise }\end{cases}
\end{aligned}
$$

where $d(i, j)$ represents the distance between the $i$-th and $j$-th residues in the model to be assessed (i.e., $M_{\text {model }}$ ).

The Estimated Quality Score Using $S_{\text {global }}$ and $S_{\text {local }}$ Models with low $S_{\text {global }}$ values are strongly estimated to be incorrect models even if the models have very high $S_{\text {local }}$. To identify models that are either too small or are incorrect and which do not have a consensus of global conformation, United3D performs a kernel density estimation (KDE) analysis. The density of models based on $S_{\text {global }}$ was estimated by Eq. 5 as follows,

$$
\begin{aligned}
& f_{h}(x)=\frac{1}{N h} \sum_{i \in N} K\left(S_{\text {global }, i}, x, h\right) \\
& K(a, b, h)=\frac{1}{\sqrt{2 \pi}} e^{-(a-b)^{2} / 2 h^{2}}
\end{aligned}
$$

where $K$ is a Gaussian function kernel, $h$ is the bandwidth $(=0.02), N$ is the number of models to be assessed, and $S_{\text {global }, i}$ is the $S_{\text {global }}$ for $i$-th model calculated using Eq. 1. We used the threshold of the first cluster where the first concave curve was detected from the KDE analysis. For example, the KDE of $S_{\text {global }}$ in the target T0498 (from CASP8) is shown in Fig. 1. The models for which $S_{\text {global }}$ was greater than or equal to 0.67 were detected as the first cluster (i.e., high quality models). The bandwidth $h$ was optimized on a training data set made 
T0498

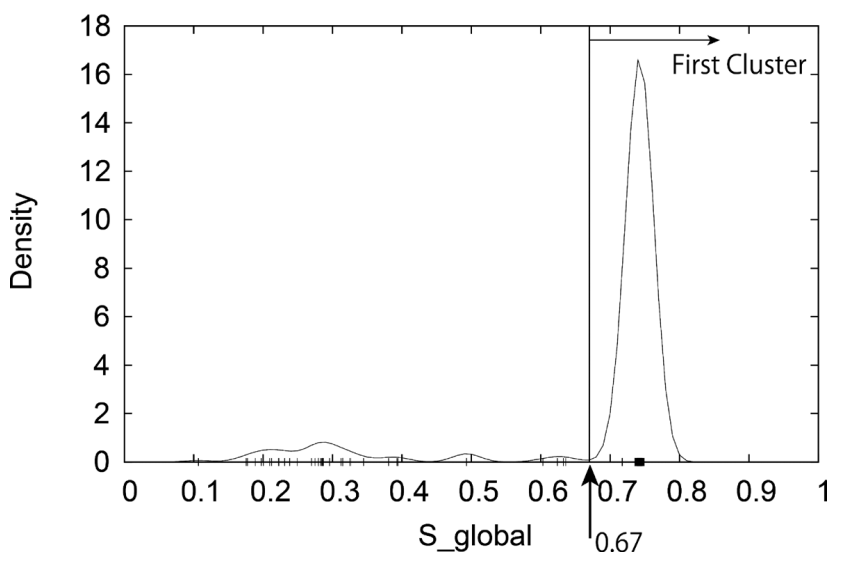

Fig. 1. Kernel Density Estimation of $S_{\text {global }}$ in the CASP8 Target T0498 S_global represents the models that correspond to the $S_{\text {global }}$ in T0498.

up of the server models from CASP8.

The Qscore can be described simply as follows,

$$
\text { Qscore }=S_{\text {global }}+w \cdot\left(S_{\text {local }}-\min \left(S_{\text {local }}^{\prime}\right)\right)
$$

where $S_{\text {local }}^{\prime}$ is a set of $S_{\text {local }}$ models from the first cluster defined by the KDE analysis, and $w$ is a weight function of the $S_{\text {local }}$ defined as follows,

$$
w= \begin{cases}0.02 & \text { if } \operatorname{STD}\left(S_{\text {global }}^{\prime}\right)<0.02 \text { and } S_{\text {global }} \geq \min \left(S_{\text {global }}^{\prime}\right) \\ 0 & \text { otherwise }\end{cases}
$$

where $\operatorname{STD}\left(S_{\text {global }}^{\prime}\right)$ represents the standard deviation of $S_{\text {global }}^{\prime}$. $S_{\text {global }}^{\prime}$ is a set of $S_{\text {global }}$ models also from the first cluster. All parameters were optimized on average Kendall's $\tau$ (described in the following section) per target data set using the server models from CASP8.

\section{Results and Discussion}

Evaluation of Qscore We evaluated the performance of United3D and other QA methods and participated in the QA category of CASP9. We compared the actual quality of models (GDT_TS) and the estimated quality score (Qscore, Eq. 6) and evaluated the performance of United3D from two points of view: (1) the correlations between the estimated quality (Qscore) and actual quality (GDT_TS) of the models; and (2) the ability to pick out the best models from the set of models that were being assessed. The former corresponds to the correlation coefficients, Pearson's $r$ and Kendall's $\tau$, and the latter is the average loss of GDT_TS ( $\triangle \mathrm{GDT})$ that is a measure of the difference between the GDT_TS of the model that was ranked top by United3D and the GDT_TS of the actual best model. Pearson's $r$ is a measure of the linear relationship between the estimated quality of model and its real quality (GDT_TS) calculated as follows,

$$
r=\frac{\sum_{i \in N}\left(Q_{i}-\bar{Q}\right)\left(\mathrm{GDT}_{i}-\overline{\mathrm{GDT}}\right)}{\sqrt{\sum_{i \in N}\left(Q_{i}-\bar{Q}\right)^{2}} \sqrt{\sum_{i \in N}\left(\mathrm{GDT}_{i}-\overline{\mathrm{GDT}}\right)^{2}}}
$$

where $N$ is the total number of target models that were evaluated by the groups in the QA category of CASP9, $Q_{i}$ is the estimated quality value of the $i$ th model, $\bar{Q}$ is the average value of the estimated quality values, $\mathrm{GDT}_{i}$ represents the GDT_TS of the $i$ th model, and $\overline{\text { GDT }}$ is the average value of GDT_TS for the evaluated models.

Kendall's $\tau$ is a measure of the probability that the model with the better estimated Qscore is actually the better model in the given random pair of models to be assessed. Kendall's $\tau$ is calculated as follows,

$$
\tau=\frac{4 \sum_{i}^{N} \sum_{i<j}^{N} P_{i, j}}{N(N-1)}-1
$$

where $P_{i, j}$ is 1 if the $i$ th or $j$ th model with the better estimated Qscore is actually the better model, otherwise it is set to zero; and $N$ represents the number of models that were assessed.

We also evaluated two naïve consensus methods, Mediand3.5 and Median GDT, as the base line. Median-d3.5 is a simple consensus method that counts only the number of residues pairs that are at distances shorter than $3.5 \AA$ after superimposing two structures. The scoring function $S_{\text {median-d3.5 }}$ is described as follows,

$$
S_{\text {median-d3.5 }}=\frac{\sum_{i \in N} \operatorname{SIM}_{3.5}\left(M_{\text {model }}, M_{i}\right)}{N}
$$

where $N$ is the number of first models predicted by each of the servers. Thus, $S_{\text {median-d3.5 }}$ represents the average structural similarity between two structures.

Median-GDT is described as follows,

$$
\begin{aligned}
& \sum_{i \in N}\left(\mathrm{SIM}_{1.0}\left(M_{\text {model }}, M_{i}\right)+\mathrm{SIM}_{2.0}\left(M_{\text {model }}, M_{i}\right)\right. \\
& S_{\text {median-GDT }}=\frac{\left.+\operatorname{SIM}_{4.0}\left(M_{\text {model }}, M_{i}\right)+\operatorname{SIM}_{8.0}\left(M_{\text {model }}, M_{i}\right)\right)}{4 N}
\end{aligned}
$$

The $S_{\text {median-GDT }}$ value described in Eq. 11 is very similar to the GDT_TS.

The 116 CASP9 Targets We compared the eight topranked QA methods in CASP9 ${ }^{9)}$ (Mufold, MuFOLDWQA, MuFOLD-QA, ${ }^{22,23)}$ QMEANClust, ${ }^{24)}$ United3D, Multicomcluster, ${ }^{25)}$ MetaMQAPclust, ${ }^{26)}$ and MQAPmulti ${ }^{27)}$ ) and the two naïve consensus methods on 116 CASP9 targets (Table 1). The top eight QA programs were defined by the CASP9 assessor according to the results of paired Student's $t$-tests. They were reported to be statistically indistinguishable from each other and to perform better than the other QA methods in the category. $\left.{ }^{9}\right)$ Interestingly, all of the top eight predictors use a clustering method to calculate scores. For the ten methods listed in Table 1, we compared the average correlation coefficients of Pearson's $r$ and Kendall's $\tau$ and the average $\Delta$ GDT among the 116 CASP9 targets and calculated the correlation coefficients of Pearson's $r$ and Kendall's $\tau$ for all quality estimations predicted by the methods in the QA category (overall). The "overall" results represent the ability of the methods to predict the absolute quality of models, which was estimated from the corresponding protein sequences.

United3D produced the best average $\Delta$ GDT (5.323) performance among the 46 QA methods and the two naïve consensus methods (Table 1). The United3D average $\triangle$ GDT was significantly better in the paired Student's $t$-test than the 
Table 1. Results of the 8 Top-Ranked and Two Naïve Consensus Methods Tested on the 116 CASP9 Targets

\begin{tabular}{|c|c|c|c|c|c|c|}
\hline & \multicolumn{2}{|c|}{ Pearson } & \multicolumn{2}{|c|}{ Kendall } & \multicolumn{2}{|c|}{$\Delta \mathrm{GDT}$} \\
\hline & Average $^{a)}$ & Overall $^{b)}$ & Average $^{c)}$ & Overall $^{d)}$ & Average $\left.^{e}\right)$ & $\#^{f}$ \\
\hline QMEANCLUST & 0.935 & 0.948 & 0.694 & 0.811 & 5.726 & 116 \\
\hline MUFOLD-QA & 0.935 & 0.933 & 0.691 & 0.792 & 6.044 & 116 \\
\hline MUFOLD-WQA & 0.934 & 0.901 & 0.694 & 0.687 & 5.503 & 116 \\
\hline UNITED3D & 0.932 & 0.935 & 0.680 & 0.784 & 5.323 & 116 \\
\hline MULTICOM-CLUSTER & 0.930 & 0.942 & 0.682 & 0.799 & 5.639 & 116 \\
\hline MUFOLD & 0.928 & 0.937 & 0.676 & 0.789 & 5.723 & 113 \\
\hline METAMQAPCLUST & 0.915 & 0.939 & 0.688 & 0.796 & 6.134 & 116 \\
\hline MQAPMULTI & 0.914 & 0.929 & 0.665 & 0.779 & 6.199 & 116 \\
\hline Median-GDT $\left.{ }^{g}\right)$ & 0.926 & 0.940 & 0.674 & 0.794 & 5.753 & 116 \\
\hline Median-MAXSUB3. $5^{h)}$ & 0.930 & 0.929 & 0.677 & 0.781 & 5.419 & 116 \\
\hline Zhang-Server $\left.{ }^{i}\right)$ & & & & & 6.660 & 116 \\
\hline
\end{tabular}

The values indicated in bold were the best among all 46 methods assessed in the QA category of CASP9. $a$ ) Average of the Pearson linear correlation coefficient per target. b) Pearson linear correlation coefficient for all quality estimations. c) Average of the Kendall rank correlation coefficient per target. $d$ ) Kendall rank correlation coefficient for all quality estimations. e) Average loss of GDT_TS between the models predicted as the best and the actually best models. $f$ ) Number of targets predicted by the groups in the QA category. $g, h$ ) Naïve consensus methods. $i$ ) Best tertiary structure prediction server for the 116 targets of CASP9.

A

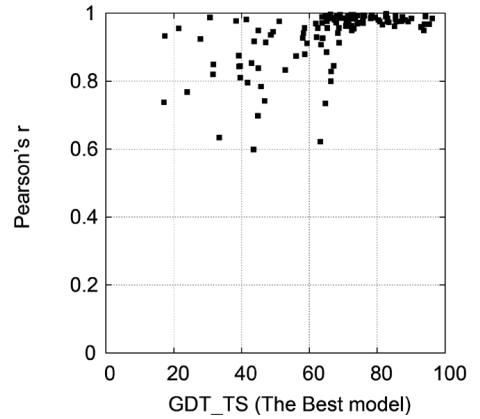

B

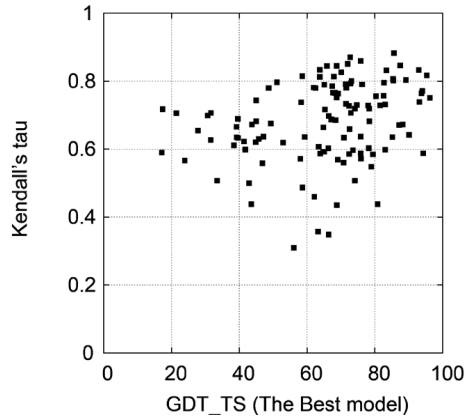

C

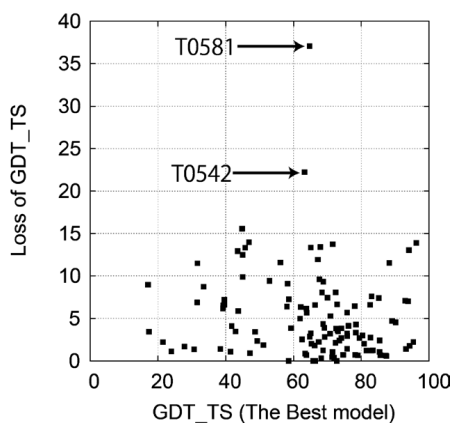

Fig. 2. Scatter Plots of the GDT TS of the Best Models versus (A) Pearson's $r$, (B) Kendall's $\tau$ and (C) Loss of GDT_TS between the Best Models and the Models Selected by United $3 \mathrm{D}$ as the Best

Zhang-Server $\left.{ }^{28}\right)(p$-value $=0.002)$ which was the best tertiary structure prediction server in CASP9. United3D was ranked in the best group for its ability to identify the best models from the models from the CASP9 servers. The performance of United3D was very similar to the performance of the topranked QA methods (QMEANCLUST, MUFOLD-QA and MUFOLD-WQA) in terms of the average correlations coefficients of Pearson's $r$ and Kendall's $\tau$. QMEANCLUST and MUFOLD-QA that had the highest average linear correlation coefficients (Pearson's $r=0.935$ ) were not significantly different from United3D (Pearson's $r=0.932$ ). Further, the difference between the average of the rank correlation coefficients for MUFOLD-QA (Kendall's $\tau=0.694$ ) and United3D (Kendall's $\tau=0.680$ ) was ignorable.

A comparison between the GDT_TS of the best models and the linear correlation coefficients for each CASP9 target is shown in scatter plots in Fig. 2A. When high quality models (GDT TS >70) were available in the set of models that were to be assessed, the Qscore predicted by United3D had very high linear correlations with the actual quality of the models (Fig. 2A). However, some of the targets that United3D showed to have particularly high linear correlations (close to 1.0) included incorrect models that had very low GDT_TS and Qscores; these incorrect models deviated dramatically from the normal distribution for the data set, which consisted of a

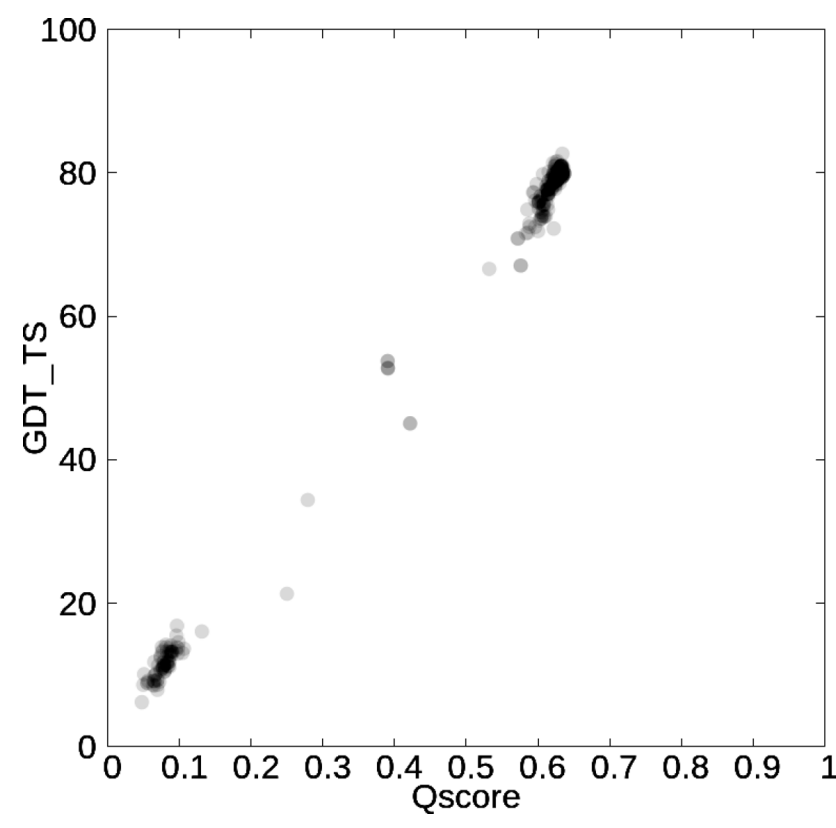

Fig. 3. Example of the Best Liner Correlations (T0638, Pearson's $r=0.998$ ) among the 116 CASP9 Targets Estimated Using United3D 

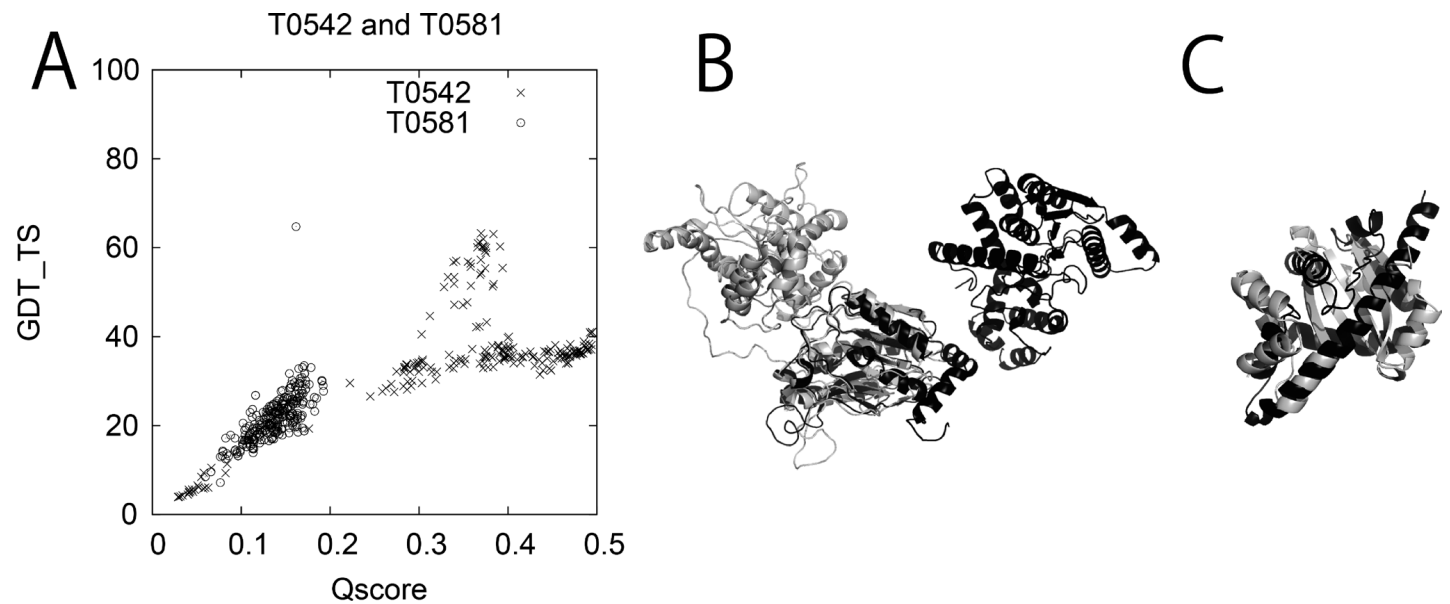

Fig. 4. (A) Scatter Plot of Qscore Estimated by United3D versus GDT_TS for the Incorrect Predictions of the Best Models of T0542 and T0581 in CASP9 and (B) the Model Predicted as Best by United3D (Black) and the Actual Best Model (Gray) in T0542 (C) The Model Predicted as Best by United3D (Black) and the Actual Best Model (Gray) in T0581

The two models were superimposed in the first domain.

Table 2. Results of the 8 Top-Ranked Methods and Two Naïve Consensus Methods Tested on the 91 Single Domain CASP9 Targets

\begin{tabular}{|c|c|c|c|c|c|c|}
\hline & \multicolumn{2}{|c|}{ Pearson } & \multicolumn{2}{|c|}{ Kendall } & \multicolumn{2}{|c|}{$\Delta \mathrm{GDT}$} \\
\hline & Average & Overall & Average & Overall & Average & $\#$ \\
\hline MUFOLD-WQA & 0.943 & 0.908 & 0.698 & 0.671 & 5.464 & 91 \\
\hline QMEANCLUST & 0.939 & 0.948 & 0.696 & 0.801 & 5.812 & 91 \\
\hline MUFOLD-QA & 0.938 & 0.930 & 0.692 & 0.779 & 6.309 & 91 \\
\hline UNITED3D & 0.937 & 0.936 & 0.684 & 0.775 & 5.357 & 91 \\
\hline MULTICOM-CLUSTER & 0.935 & 0.939 & 0.686 & 0.787 & 5.733 & 91 \\
\hline MQAPMULTI & 0.935 & 0.929 & 0.682 & 0.769 & 6.221 & 91 \\
\hline MUFOLD & 0.932 & 0.935 & 0.678 & 0.777 & 5.817 & 88 \\
\hline METAMQAPCLUST & 0.929 & 0.947 & 0.698 & 0.802 & 6.145 & 91 \\
\hline Median-GDT & 0.931 & 0.937 & 0.679 & 0.781 & 5.818 & 91 \\
\hline Median-MAXSUB3.5 & 0.937 & 0.931 & 0.681 & 0.774 & 5.486 & 91 \\
\hline QUARK $^{a}$ ) & & & & & 6.790 & 91 \\
\hline
\end{tabular}

a) Best tertiary structure prediction server for the 91 single domain CASP9 targets.

majority of high quality models. Figure 3 shows, for example, a comparison between the estimated quality and the GDT_TS for T0638, which United3D estimated to have the highest Pearson's $r$ (0.998) among the 116 targets. Only two clusters consisting of both very accurate models and incorrect models were found; therefore, although the Pearson's $r$ was almost 1.0, this result was unusual (Fig. 3). Thus, we might need to consider that for high Pearson's $r$ values close to 1.0, the results do not truly represent the real performance of all of the top ranked QA methods.

On the other hand, the GDT_TS of the best models do not have a clear relationship with the rank correlation coefficients (Fig. 2B) or with the ability of United3D to select the best model (Fig. 2C). Figures $2 \mathrm{~B}$ and $\mathrm{C}$ show that, unlike Pearson's $r$, Kendall's $\tau$ and $\Delta$ GDT do not strongly depend on the distribution of the actual quality of models. These results also indicate that United3D can constantly distinguish between high quality models and incorrect models, whether or not high quality models are available in the data set.

The two significant worse quality estimations were for T0542 and T0581 (Fig. 2C). Although the best models were of medium quality (GDT_TS $=63.2,64.7)$, the worst estimations were for the T0542 and T0581 models that had poor GDT_TS (41.0, 27.7, respectively) but were ranked as the best by United3D. Scatter plots of the Qscore estimated by United3D versus the GDT_TS for T0542 and T0581 are shown in Fig. 4A. The native structure of T0542 consists of two domains and the models that were assessed had various relative positions for the first and second domains. As shown in Fig. 4B, the model ranked as the best by United3D has a different relative position for the two domains with the actual best model. Thus, the actual quality (GDT_TS) of the models is dependent, not only on the conformation of each domain, but also on the relative arrangement of the domains. This dependency is a well-known partial limitation of the evaluation methods that were tested in the QA category. When the structures of the models were split into two subunits, one for each domain regions, the GDT_TS for the models that were ranked as best by United3D were 66.3 and 86.6 (loss of GDT_TS was only 3.7 and 0.0 ) for the first and second domains, respectively. For the other model (T0581) with the worst estimates, only one relatively high-quality model (GDT_TS=64.7) stood out. This model was different from the other models in the cluster (i.e., far from the centroid of cluster). In Fig. 4C, the actual 

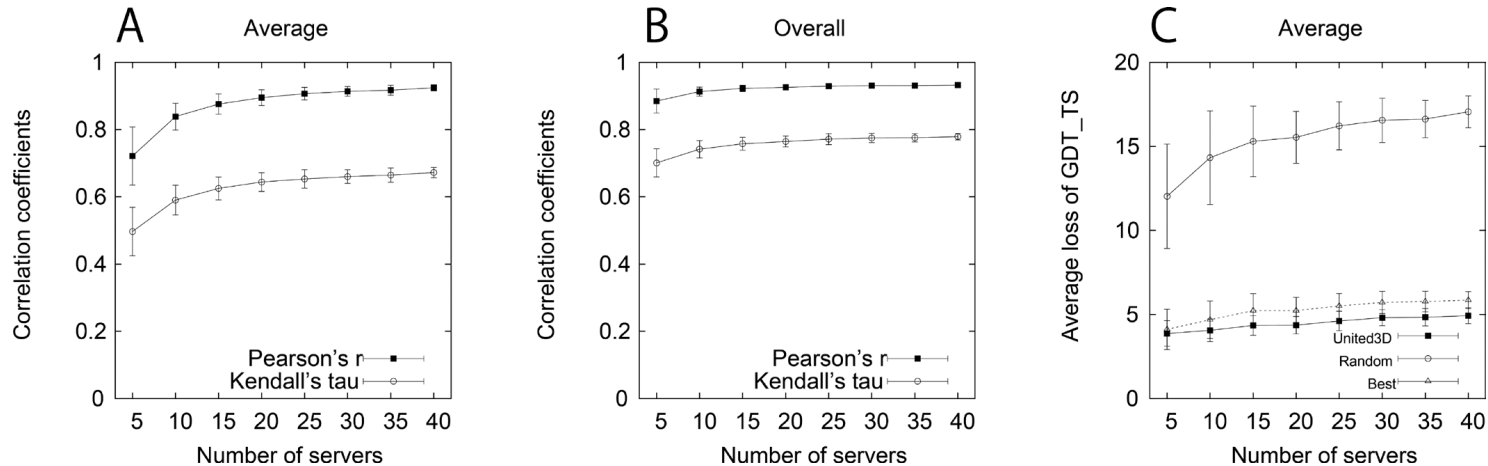

Fig. 5. Number of Servers Used in the United3D Method versus the Performance of United3D for the 116 CASP9 Targets

The error bars denote the standard deviations of the values calculated from each plots for the 100 data sets. (A) Average Pearson's $r$ and Kendal's $\tau$ for the 116 targets. (B) Pearson's $r$ and Kendal's $\tau$ for the "overall" models. (C) Average loss of GDT_TS among the 116 targets. Random represents "random server," the average results for the randomly selected servers; Best represents "best server," the best individual tertiary structure prediction server from among the randomly selected servers; and United3D represents the average loss of the GDT_TS between the best model in the data set and the best models selected by United3D.

best model has clearly different fold from the model ranked as best by United3D. Because of limitations in the clustering methods, United3D cannot identify accurate models that are situated far from the centroid of a cluster in a data set. Therefore, the use of another type of scoring function, such as a knowledge-based scoring function, should improve United3D which currently uses only clustering methods.

Some of the targets in CASP9 contained several domains that, in nature, could assume various relative arrangements. To avoid the influence of different possible domain orientations on the results, we separately evaluated the results of the QA methods for single domain targets. Table 2 shows the results of the eight top-ranked QA methods in the CASP9 QA category, the two naïve consensus methods and the best server (QUARK) ${ }^{28)}$ for 91 single-domain targets. The results were similar to those shown in Table 1. United3D had the best average $\triangle \mathrm{GDT}$ and correlation coefficients that were very similar to the best QA methods (MUFOLD-WQA and METAMQAPCLUST).

Real Application of United3D to Assess the Quality of Predicted 3D Structures Clustering methods estimate the quality of models based on their distances from the cluster centroids. Therefore these methods require enough predicted protein models using different prediction methods. But how many individual models are needed for the accurate quality estimation by United3D can be difficult to determine. To address this limitation, we ran United3D using a set of models that was obtained from a limited number of servers. Figure 5 shows the average results for 100 data sets containing models that were generated using from 5 to 40 servers randomly selected from the 78 servers that participated in CASP9. A 5-steps procedure was employed: (1) the number of servers $(N)$ was set to 5 ; (2) $N$ servers were selected from the 78 servers in CASP9; (3) United3D was run on the subset of models that were predicted for the 116 targets by the servers selected in step (2); (4) the results from step (3) were evaluated by comparing them with the actual quality of the selected models (GDT_TS); and (5) steps (2)-(4) were repeated 100 times. Then, steps (2)-(5) were repeated for different values of $N$ (5, $10,15,20,25,30,35,40)$.

The results in Fig. 5A show that the linear and rank correlation coefficients were both dependent on the number of servers that were used to generate the models. When a small number of servers (5-10) were used, the performance of United3D for the average correlation per target was not good. The correlations for the "overall" models (Fig. 5B) that correspond to the performance of United3D for the estimation of absolute quality were also dependent on the number of available servers, but the effect was quite limited. On the other hand, the United3D results for the average loss of GDT_TS did not depend on the number of servers that were used (Fig. 5C). We also evaluated the performance of a "random server" and a "best server." The "random server" was the server that had an average performance for the set of models that were assessed; the "best server" was the server that had the best performance of all the servers selected in step (2). United3D performed significantly better performance than the "random server" based on the results of the paired Student's $t$-test ( $p$-value $<0.05)$, and United3D performed significantly better statistically than the "best server" for all values of $N(5-40)$ (Fig. 5C). The goal of United3D is to select the models that are as good as the best models from a set of available models. We have shown that, on average, when at least 5 individual servers are available, United3D can consistently select models that are better than the models selected by the best available server.

\section{Conclusion}

Here, we describe a QA method, United3D that we have developed and tested. United3D can identify near-best models using two types of clustering methods.

In the QA category of CASP9, United3D was ranked 1st in selecting the actual high quality models from the set of models predicted by CASP9 servers. Moreover, Qscore of United3D showed very high correlations with the actual quality of models (GDT_TS), and was found to be competitive with the other top-performing QA methods in the category. The linear correlations were very high (0.93) and the rank correlations were acceptable (0.68). In addition, the quality of models selected by United3D was 5\% lower than the best available models on average. These results indicate that the performance of United3D in estimating the quality of very similar models can be further improved.

Our evaluation of possible real applications revealed that United3D can select models that are better than the models selected by the best available server when the number of available individual servers varies between a large number and 
a very limited number (five). Thus, the ability of United3D to select the best models does not depend on the number of available individual servers that provide the data set of predicted models.

Our results indicate that United3D will be very useful for selecting the best models from a set of models provided by a variety of individual methods and the Qscore of United3D will lead to further improvements in protein structure prediction.

\section{References}

1) Moult J., Fidelis K., Kryshtafovych A., Tramontano A., Proteins, 79 (Suppl. 10), 1-5 (2011).

2) 9th Community Wide Experiment on the Critical Assessment of Techniques for Protein Structure Prediction. "CASP9 Experiment.": 〈http://predictioncenter.org/casp9/,, cited 14 April, 2010.

3) Tress M., Ezkurdia I., Graña O., López G., Valencia A., Proteins, 61 (Suppl. 7), 27-45 (2005).

4) Kopp J., Bordoli L., Battey J. N., Kiefer F., Schwede T., Proteins, 69, (Suppl. 8), 38-56 (2207).

5) Cozzetto D., Kryshtafovych A., Fidelis K., Moult J., Rost B., Tramontano A., Proteins, 77 (Suppl. 9), 18-28 (2009).

6) Mariani V., Kiefer F., Schmidt T., Haas J., Schwede T., Proteins, 79 (Suppl. 10), 37-58 (2011).

7) Cozzetto D., Kryshtafovych A., Ceriani M., Tramontano A., Proteins, 69 (Suppl. 8), 175-183 (2007).

8) Cozzetto D., Kryshtafovych A., Tramontano A., Proteins, 77 (Suppl. 9), 157-166 (2009).

9) Kryshtafovych A., Fidelis K., Tramontano A., Proteins, 79 (Suppl.
10), 91-106 (2011).

10) Eisenberg D., Lüthy R., Bowie J. U., Methods Enzymol., 277, 396404 (1997).

11) Zhou H., Zhou Y., Protein Sci., 11, 2714-2726 (2002).

12) Tosatto S. C., J. Comput. Biol., 12, 1316-1327 (2005).

13) Pettitt C. S., McGuffin L. J., Jones D. T., Bioinformatics, 21, 3509_ 3515 (2005).

14) Lee M. R., Tsai J., Baker D., Kollman P. A., J. Mol. Biol., 313, 417-430 (2001)

15) Ginalski K., Elofsson A., Fischer D., Rychlewski L., Bioinformatics, 19, 1015-1018 (2003).

16) Zhang Y., Skolnick J., J. Comput. Chem., 25, 865-871 (2004).

17) Zhou H., Skolnick J., Proteins, 71, 1211-1218 (2008).

18) Wallner B., Elofsson A., Proteins, 69 (Suppl. 8), 184-193 (2007).

19) Larsson P., Skwark M. J., Wallner B., Elofsson A., Proteins, 77 (Suppl. 9), 167-172 (2009).

20) McGuffin L. J., Proteins, 77 (Suppl. 9), 185-190 (2009).

21) Archie J. G., Paluszewski M., Karplus K., Proteins, 77 (Suppl. 9), 191-195 (2009).

22) Zhang J., Wang Q., Barz B., He Z., Kosztin I., Shang Y., Xu D., Proteins, 78, 1137-1152 (2010).

23) Wang Q., Vantasin K., Xu D., Shang Y., Proteins, 79 (Suppl. 10), 185-195 (2011).

24) Benkert P., Schwede T., Tosatto S. C., BMC Struct. Biol., 9, 35 (2009).

25) Wang Z., Eickholt J., Cheng J., Bioinformatics, 26, 882-888 (2010).

26) Pawlowski M., Gajda M. J., Matlak R., Bujnicki J. M., BMC Bioinformatics, 9, 403 (2008).

27) Wang Z., Cheng J., Protein Sci., 21, 142-151 (2012).

28) Xu D., Zhang J., Roy A., Proteins, 79 (Suppl. 10), 147-160 (2011). 